\title{
Multifractal structure of the large-scale heliospheric magnetic field strength fluctuations near $85 \mathrm{AU}$
}

\author{
L. F. Burlaga \\ NASA-Goddard Space Flight Center, Laboratory for Extraterrestrial Physics, Code 692, Greenbelt, Maryland 20771, USA
}

Received: 22 March 2004 - Revised: 26 May 2004 - Accepted: 3 June 2004 - Published: 28 September 2004

\begin{abstract}
During 2002, the Voyager 1 spacecraft was in the heliosphere between 83.4 and 85.9 AU (1 AU is the mean distance from the Sun to Earth) at $34^{\circ} \mathrm{N}$ heliographic latitude. The magnetic field strength profile observed in this region had a multifractal structure in the range of scales from 2 to 16 days. The multifractal spectrum observed near $85 \mathrm{AU}$ is similar to that observed near $40 \mathrm{AU}$, indicating relatively little evolution of the multifractal structure of the magnetic field with increasing distance in the distant heliosphere in the epoch near solar maximum.
\end{abstract}

\section{Introduction}

The Sun is surrounded by a region called the "heliosphere" whose radial extent is at least $85 \mathrm{AU}$, which is filled with plasma and magnetic fields moving from the Sun toward the interstellar medium at an average speed of $\approx 450 \mathrm{~km} / \mathrm{s}$. This "solar wind" is supersonic relative to the characteristic speeds of the medium. The plasmas and magnetic fields in the heliosphere have a variety of sources on the Sun and they move with a broad range of speeds, so that they interact as they move away from the Sun. The diversity of the flows and their interactions on a wide range of scales lead to a complex structure of the heliospheric magnetic field which is observed in "large-scale fluctuatuations" that are seen on scales from $\approx 1$ day to a few solar rotations $(\approx 75$ days $)$ in plots of the data on a scale of the order of 1 year (Burlaga, 1995).

Multifractal structure of the magnetic field and velocity has been observed on small scales (a few seconds to a few hours) in relation to intermittent turbulence in the solar wind observed at $<5 \mathrm{AU}$. Intermittent turbulence has been the subject of many studies, and it continues to be an active area of research (Burlaga, 1991; Milovanov and Zelenyi, 1994;

Correspondence to: L. F. Burlaga

(leonard.f.burlaga@nasa.gov)
Marsch and Liu, 1993; Marsch and Tu, 1996, 1997; Horbury et al., 1997; Carbone and Bruno, 1997; and Bruno et al., 2003). However, this paper discusses multifractal structure observed on much larger scales and distances. Although the methods used to study the multifractal structure of turbulence can be used to study the multifractal structure of the large-scale fluctuations (Burlaga, 1995), the latter represent a different physical phenomenon, related to the interactions among streams, ejecta, interaction regions, discontinuities and shocks, etc. These large-scale fluctuations might act as sources of the small-scale turbulence, and in some respects they are mathematically similar to turbulence, but they represent different processes.

Multifractal structure of the large-scale fluctuations in magnetic field strength data from Voyager 2 near $25 \mathrm{AU}$ was observed on scales from $16 \mathrm{~h}$ to 21 days (Burlaga et al., 1991). Multifractal structure of B was observed at other distances and at three phases of the solar cycle (the declining phase, solar minimum, and the rising phase approaching solar maximum) (Burlaga et al., 1993). The structure of the heliospheric magnetic field strength observed near $40 \mathrm{AU}$ by Voyager 1 (V1) was multifractal, and this structure can be explained by a 1-D multi-fluid MHD model of the solar wind with observations at $1 \mathrm{AU}$ as input to the model (Burlaga et al., 2003b).

The magnetic field is so weak beyond $40 \mathrm{AU}$ that it has not been possible until now to determine whether the multifractal structure persists beyond $40 \mathrm{AU}$. Exceptionally high quality magnetic field data were obtained by V1 during 2002 . We use 24-hour averages of these data to show that in 2002 largescale fluctuations in the heliospheric magnetic field strength had a multifractal structure at $\approx 85 \mathrm{AU}$, near the termination shock (McDonald et al., 2003; Krimigis et al., 2003). Thus, the multifractal structure of the large-scale fluctuations in the magnetic field strength is a universal property of the heliosphere, extending from 1 to $85 \mathrm{AU}$. 


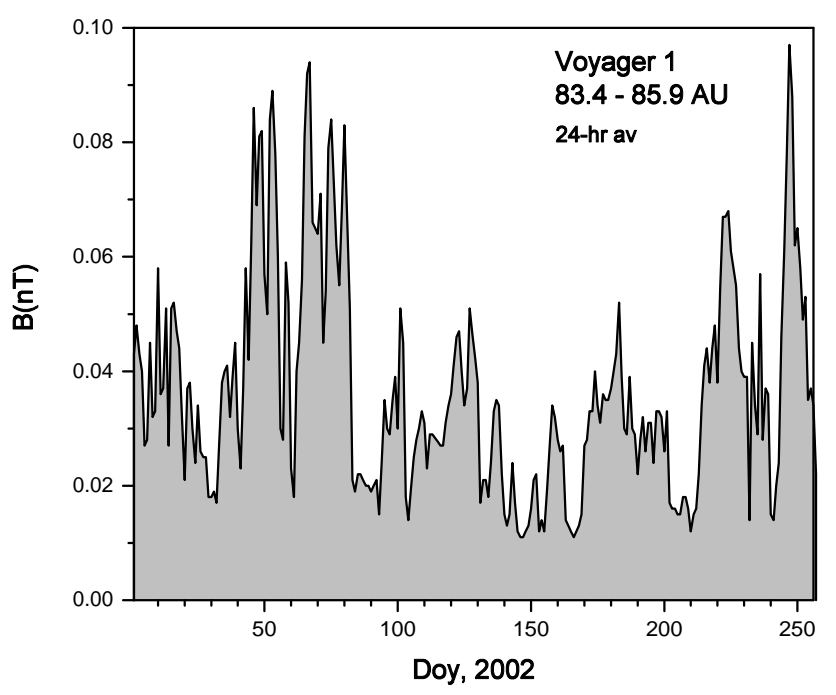

Fig. 1. Daily averages of the magnetic field strength as a function of time observed by V1 near 85 AU from day 1 to 256, 2002.

\section{Multifractal distributions}

The basic theory of multifractals has been discussed in several papers (Frisch et al., 1978; Mandelbrot, 1972; Stanley and Meakin, 1988; Tel, 1988). Given a time series B(t), normalized by the average value of the magnetic field strength in the interval under consideration, one examines averages $<\mathrm{B}_{n}^{q}>$ of the qth moments of $\mathrm{B}$ on various time scales $\tau_{n}=2^{n}$, where $\mathrm{n}$ has the values $0,1,2 \ldots ; \tau_{o}=1$ day in this paper. If the magnetic field strength profile has a multifractal structure, then

$<\mathrm{B}_{\mathrm{n}}^{\mathrm{q}}>\sim \tau_{\mathrm{n}}^{\mathrm{s}(\mathrm{q})}$

over some range of scales (see Burlaga, 1995, page 193).

In practice, the function $\mathrm{s}(\mathrm{q})$ is determined by fitting a loworder polynomial to a set of observations (q, s) over some range of scales for integers $q$ between -10 and 10. Thus, just a few numbers (the coefficients of the polynomial) describe the multifractal. For a time series whose distribution of values is lognormal, $\mathrm{s}(\mathrm{q})$ is a quadratic polynomial (Mandelbrot, 1972). The results that we shall present can be fit by a cubic polynomial, within the uncertainties of the observations:

$\mathrm{s}(\mathrm{q})=\mathrm{a}_{0}+\mathrm{a}_{1} \mathrm{q}+\mathrm{a}_{2} \mathrm{q}^{2}+\mathrm{a}_{3} \mathrm{q}^{3}$

This cubic fit to the observations provides a higher order description of the scaling symmetry than a lognormal distribution function (see Burlaga, 2001 and references therein). It constrains any model that might be proposed to explain the observations. However, the cubic polynomial is not a unique fitting function and it is not a solution of a physical equation; it is simply a convenient and useful way to summarize the observations of $\mathrm{s}(\mathrm{q})$.

Multifractal data are most directly described by the slopes $\mathrm{s}(\mathrm{q})$, representing the scaling properties of fluctuations with

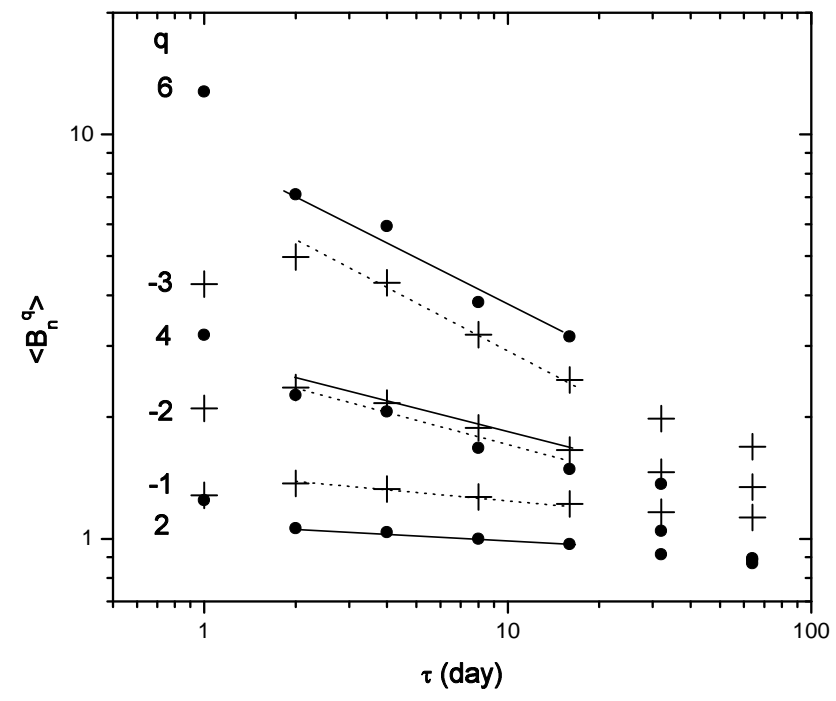

Fig. 2. The qth moments of various averages of $B^{q}$ as a function of scale for selected values of $q$. There is a range of scales in which the points for a given moment $q$ lie close to a straight line. The absolute value of the slope of a line increases with increasing q, indicating the existence of multifractal structure.

different amplitudes. Two other descriptions have been introduced. The first is the "generalized dimension" $\mathrm{D}_{\mathrm{q}}(\mathrm{q})$, (Hentschel and Procaccia, 1983; Sreenivasan, 1991) which is related to $\mathrm{s}(\mathrm{q})$ by the equation

$\mathrm{D}_{\mathrm{q}}(\mathrm{q})=1+\mathrm{s}(\mathrm{q}) /(\mathrm{q}-1)$

The second description is in terms of the "multifractal spectrum" $\mathrm{f}(\alpha)$ (Halsey et al., 1986; Sreenivasan, 1991; Stanley and Meakin, 1988; Tel, 1988) defined by the relations

$\alpha=\mathrm{d} / \mathrm{dq}\left[(\mathrm{q}-1) \mathrm{D}_{\mathrm{q}}(\mathrm{q})\right]$

$\mathrm{f}(\alpha)=\mathrm{q} \alpha(\mathrm{q})-(\mathrm{q}-1) \mathrm{D}_{\mathrm{q}}(\mathrm{q})$

In the particular case where $\mathrm{s}(\mathrm{q})$ is a cubic polynomial within the measurement uncertainties, the preceding equations give

$\alpha=1+\mathrm{a}_{1}+2 \mathrm{a}_{2} \mathrm{q}+3 \mathrm{a}_{3} \mathrm{q}^{2}$

$\mathrm{f}(\alpha)=1-\mathrm{a}_{0}+\mathrm{a}_{2} \mathrm{q}^{2}+2 \mathrm{a}_{3} \mathrm{q}^{3}$

The polynomial forms of $\alpha$ and $\mathrm{f}(\alpha)$ are consequences of the choice of a cubic polynomial describing the observations of $\mathrm{s}$ versus $\mathrm{q}$. The coefficients in the polynomials are not unique, and they do not have direct physical significance. However, these coefficients do give estimates of the points at which $\mathrm{f}(\alpha)=0$ and the shape of $\mathrm{f}(\alpha)$, which are important properties of the multifractal structure. The parameter $\alpha$ has a critical point at $\mathrm{q}_{\max }=-\mathrm{a}_{2} /\left(3 \mathrm{a}_{3}\right)$; one cannot compute $\mathrm{f}(\alpha)$ for larger values of $\mathrm{q}$, so $\mathrm{q}_{\max }$ gives a limit on the values of $\mathrm{q}$ that can be used in the analysis. This upper value for $\mathrm{q}$ is a consequence of the limitations of the data, both the relatively small number of days in the data set and the uncertainties of the measurements. 


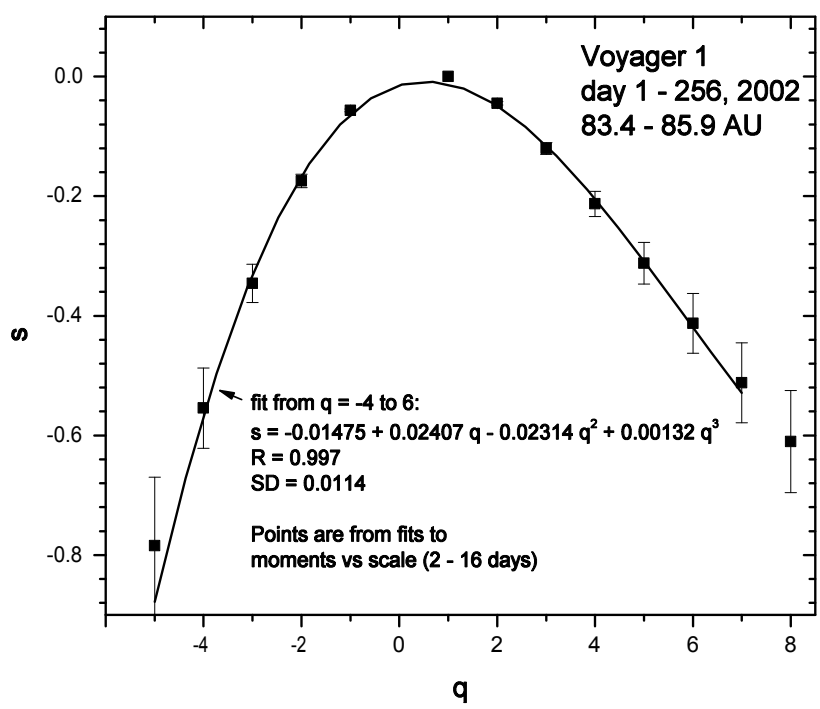

Fig. 3. The points $(\mathrm{q}, \mathrm{s})$ and their error bars derived from the observations as described in the text. The solid curve is a fit to these points with the cubic polynomial, whose equation is shown in the figure.

\section{Observations}

Figure 1 shows $\mathrm{V} 1$ observations of the daily averages of the magnetic field strength B from day 1 to 256, 2002.

During this interval V1 moved from 83.4 to $85.9 \mathrm{AU}$ at $34^{\circ}$ North heliographic latitude. The $1 \sigma$ uncertainties in the measurements are exceptionally low, $<0.015 \mathrm{nT}$, primarily owing to small changes in the spacecraft magnetic field which is the major source of error. The fluctuations in B are very large during this interval, and one can identify clusters of strong fields on various scales. In order to search for multifractal structure in this time profile, we plot $\left\langle\mathrm{B}_{n}^{q}\right\rangle$ versus $\tau_{n}$ in units of days on a $\log$ - $\log$ scale for $1 \leq \tau_{n} \leq 64$ days, i.e., for $\tau_{n}=2^{n}$ $(\mathrm{n}=0,1,2,3,4,5$, and 6$)$. We computed one curve (one set of points) for each value of $\mathrm{q}$, where $\mathrm{q}$ is an integer. For our data, scaling (power law) behavior is observed for scales $2 \leq \tau_{n} \leq 16$ days and for $-6 \leq \mathrm{q} \leq 10$, demonstrating that there is multifractal structure over this range of scales.

Using a linear least squares fitting procedure, we fit a straight line to each set of points corresponding to an integer q, on a log-log plot of $<\mathrm{B}_{n}^{q}>$ vs. $\tau_{n}$, and we obtained a slope $\mathrm{s}$ and an error bar for $\mathrm{s}$ for each set. This procedure is illustrated for $\mathrm{q}=-3,-2,-1,2,4$, and 6 in Fig. 2.

The points (q, s) and error bars obtained in this way are plotted in Fig. 3. A cubic polynomial for $q$ between -4 and 6, shown by the solid curve and equation in Fig. 3, provides a good fit to the points, and it a useful but somewhat arbitrary way to summarize and interpolate the observations. The polynomial has an inflection point at $\mathrm{q}=5.84$ owing to the uncertainties in the measurements, which sets an upper limit for the meaningful range of q. Within the measurement uncertainties, the multifractal structure of the large-scale magnetic field strength fluctuations observed by V1 near $85 \mathrm{AU}$ can be

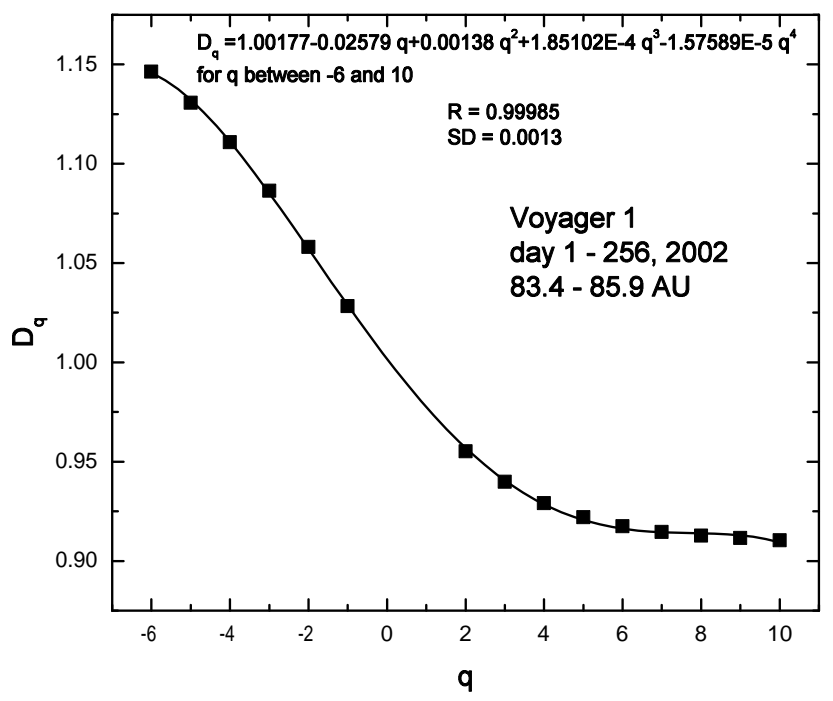

Fig. 4. The generalized dimension $D_{q}(q)$ versus $q$ computed from the points in Fig. 3. The curve is a linear least squares fit of a fourth order polynomial (show in the figure) to the points.

described by four constants, the coefficients in a cubic polynomial, s(q).

Given (q, s) derived from the observations, one can calculate corresponding values $\left(\mathrm{q}, \mathrm{D}_{\mathrm{q}}\right)$, where $\mathrm{D}_{\mathrm{q}}$ is defined in the previous section. The points $\left(\mathrm{q}, \mathrm{D}_{\mathrm{q}}\right)$ for $-6 \leq \mathrm{q} \leq 10$ are plotted in Fig. 4. One can fit these points with a fourth order polynomial, which is shown by the solid curve and the equation in Fig. 4. From the coefficients of the measured cubic polynomial $\mathrm{s}(\mathrm{q})$, which are shown in Fig. 3, one can compute a set of points in the multifractal spectrum $\mathrm{f}(\alpha)$ using the last two equations in the previous section. These points are plotted as the solid squares in Fig. 5. From the two values of $\alpha$ at which $\mathrm{f}(\alpha)=0$, one could reconstruct a time series with the same multifractal spectrum (Burlaga et al., 1993). The set of points in Fig. 5 was fitted with the cubic polynomial shown by the curve in Fig. 5. This polynomial $\mathrm{f}(\alpha)$ describes the multifractal spectrum for the V1 observations shown in Fig. 1. Note that $\mathrm{f}(\alpha)=0$ at $\alpha=0.77$, which is very close to the value of the corresponding $\alpha_{\min }$ observed near $40 \mathrm{AU}$ close to the previous maximum in solar activity (Burlaga et al., 2003b). The cubic approximation for $\mathrm{f}(\alpha)$ does not give a corresponding $\alpha_{\max }$ at which $\mathrm{f}(\alpha)=0$, owing to the presence of an inflection point. But extrapolating the slope of $\mathrm{f}(\alpha)$ linearly between the inflection point and the $\alpha$ axis gives $\alpha_{\max } \approx 1.425$, again very close that observed near $40 \mathrm{AU}$. These results suggest that there is little evolution in the multifractal structure of the heliospheric magnetic field between 40 and $85 \mathrm{AU}$ when the Sun is active.

\section{Summary and discussion}

The magnetic field strength as a function of time observed by V1 from 83.4 to $85.9 \mathrm{AU}$ during 2002 has a multifractal 


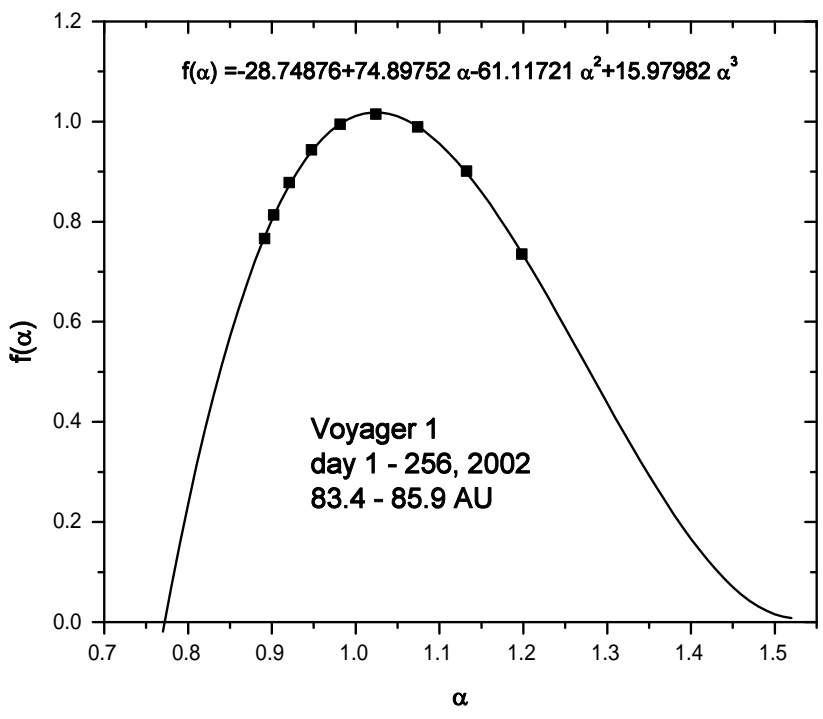

Fig. 5. The multifractal spectrum derived from the V1 observations near $85 \mathrm{AU}$ (solid squares). The solid curve is a fit to these points, a cubic polynomial whose equation is shown in the figure.

structure in the range of scales from 2 to 16 days. During 2002 , solar activity was declining from its maximum value in 2000. Since it takes nearly a year for the solar wind to propagate from the Sun to $85 \mathrm{AU}$, V1 was observing conditions determined by relatively high solar activity during 2001. We found that the multifractal structure of the heliospheric magnetic field can extend as far as $85 \mathrm{AU}$ from the Sun when the Sun is active. We also found that the multifractal spectrum of the fluctuations in B observed near $85 \mathrm{AU}$ (near the outer boundary of the solar wind) is very similar to that observed near $40 \mathrm{AU}$ in 1989 , when the solar activity was high in the previous solar cycle. Thus, there was relatively little evolution of the multifractal spectrum between 40 and $85 \mathrm{AU}$, probably because V1 was so far from the energy source, the Sun.

The existence of multifractal structure in the heliospheric magnetic field at $40 \mathrm{AU}$ can be explained by a deterministic MHD model (Burlaga et al., 2003a). The model (Wang and Richardson, 2001; Wang et al., 2000) is a time-dependent, 1-D, spherically symmetric, multi-fluid that includes pickup ions produced by the interaction of the solar wind with interstellar neutral hydrogen; the dynamical processes are driven by changes in momentum flux, gradients in the magnetic pressure, and gradients in the pressures of the solar wind protons and the pickup protons. Three-dimensional turbulence is not described by the model. In this model, the multifractal structure of the large-scale fluctuations of the magnetic field strength and speed arises from both the complexity of the initial conditions at $1 \mathrm{AU}$ (Burlaga, 1992) and from non-linear interactions among complex flows, interaction regions, and shocks between 1 and $\approx 85 \mathrm{AU}$.

The similarity of the multifractal spectrum at $\approx 85 \mathrm{AU}$ to that at $\approx 40 \mathrm{AU}$ is consistent with the predictions of the MHD model of solar wind flow through the heliosphere near solar maximum (Burlaga et al., 2003a, 2003b). This model shows that the heliospheric structure changes rapidly between 1 and $\approx 30 \mathrm{AU}$, but it changes relatively slowly in the distant heliosphere between $\approx 40$ and $90 \mathrm{AU}$. There is considerable restructuring and formation of larger scale structures between 1 and $\approx 30 \mathrm{AU}$, driven by the large gradients in momentum flux and pressure there. However, the processes involved in the restructuring react back on the flows, diminishing these gradients, so that they are no longer large enough to produce major changes between $\approx 40$ and $90 \mathrm{AU}$.

Acknowledgements. The author thanks S. Kramer and T. McClanahan for programming support, and M. Acuña for his continued assistance in evaluating the measurements. The Principal Investigator of the magnetic field experiment on Voyager 1 is N. F. Ness.

Edited by: J. Büchner

Reviewed by: two referees

\section{References}

Bruno, R., Carbone, V., Sorriso-Valvo, L., and Bavassano, B.: Radial evolution of solar wind intermittency in the inner heliosphere, J. Geophys. Res. S. P., 108 (A3), doi:10.1029/2002JA009615, 2003.

Burlaga, L. F.: Intermittent turbulence in the solar wind, J. Geophys. Res. S. P., 96 (A4), 5847-5851, 1991.

Burlaga L. F.: Multifractal structure of the magnetic field and plasma in recurrent streams at 1 AU, J. Geophys. Res., 97, 42834293, 1992.

Burlaga, L. F.: Interplanetary Magnetohydrodynamics, Oxford University Press, New York, 1995.

Burlaga, L. F.: Lognormal and multifractal distributions of the heliospheric magnetic field, J. Geophys. Res., 106, 15917-15927, 2001.

Burlaga, L. F., McDonald, F. B., Ness, N. F., and Lazarus, A. J.: Multifractal structure of the interplanetary magnetic field: Voyager 2 observations near 25 AU, 1987-1988, J. Geophys. Res., 96, 3789-3799, 1991.

Burlaga, L. F., Perko, J., and Pirraglia, J.: Cosmic ray modulation, merged interaction regions, and multifractals, Astrophys. J., 407, 347-358, 1993.

Burlaga, L. F., Wang, C., Richardson, J. D., and Ness, N. F.: Largescale magnetic field fluctuations and development of the 19992000 GMIR: 1 to 60 AU, Astrophys. J., 585, 1158-1168, 2003 a.

Burlaga, L. F., Wang, C., and Ness, N. F.: A model and observations of the multifractal spectrum of the heliospheric magnetic field strength fluctuations near $40 \mathrm{AU}$, Geophys. Res. Lett., 30, 501504, 2003b.

Carbone, V. and Bruno, R.: High-order velocity structure functions and anomalous scaling laws in the interplanetary space, Nuovo Cimento Della Societa Italiana Di Fisica C-Geophysics and Space Physics, 20 (6), 933-938, 1997.

Frisch U., Sulem, P.-L., and Nelkin, M.: A simple dynamical model of intermittent fully developed turbulence, J. Fluid Mech., 87, 719, 1978.

Halsey, T. C., Jensen, M. H., Kadanoff, L. P., Procaccia, I., and Shraiman, B. I.: Fractal measures and their singularities: The characterization of strange sets, Phys. Rev. A, 33(2), 1141-1151, 1986. 
Hentschel, H. G. E. and Procaccia, I.: The infinite number of generalized dimensions of fractals and strange attractors, Physica D: Nonlinear Phenomena 8, Issue 3, 435-444, 1983.

Horbury, T. S., Balogh, A., Forsyth, R. J., and Smith, E. J.: Ulysses observations of intermittent heliospheric turbulence, Advances in Space Research, 847-850, 1997.

Krimigis, S. M., Decker, R. B., Hill, M. E., Armstrong, T. P., Gloeckler, G., Hamilton, D. C., Lanzerotti, L. J., and Roelof, E. C.: Evidence that Voyager- 1 exited the solar wind at $\sim 85 \mathrm{AU}$, Nature, 426 (6962), 45-48, 2003.

Mandelbrot, B.: Possible refinement of the lognormal hypothesis concerning the distribution of energy dissipation in intermittent turbulence, in: Statistical Models and Turbulence, edited by Rosenblatt, M. and Van Atta, C., Springer-Verlag, BerlinHeidelberg, 333, 1972.

Marsch, E., and Liu, S.: Structure and intermittency of velocity fluctuations in the inner solar wind, Ann. Geoph., 11 (4), 227238, 1993.

Marsch, E., and Tu, C. Y.: Spatial evolution of the magnetic field spectral exponent in the solar wind: Helios and Ulysses comparison, J. Geophys. Res. S. P., 101 (A5), 11 149-11 152, 1996.

Marsch, E. and Tu, C.-Y.: Intermittency, non-Gaussian statistics and fractal scaling of MHD fluctuations in the solar wind, Nonl. Proc. Geophys., 4, 101-124, 1997,

SRef-ID: 1607-7946/npg/1997-4-101.
McDonald, F. B., Stone, E. C., Cummings, A. C., Heikkila, B., Lal, N., and Webber, W. R.: Enhancements of energetic particles near the heliospheric termination shock, Nature, 426 (6962), 4851, 2003.

Milovanov, A. and Zelenyi, P.: Development of fractal structure in the solar wind and distribution of magnetic field in the photosphere: Solar System Plasmas in Space and Time, AGU Geophys. Monograph, 84, Washington D.C., 43-52, 1994.

Sreenivasan, K. R.: Fractals and multifractals in fluid turbulence, Annu. Rev. Fluid Mech., 23, 539, 1991.

Stanley, H. E. and Meakin, P.: Multifractal phenomena in physics and chemistry, Nature, 335, 405, 1988.

Tel, T.: Fractals, multifractals, and thermodynamics, Z. Naturforsch 43a, 1154, 1988.

Wang, C. and Richardson, J. D.: Energy partition between solar wind protons and pickup ions in the distant heliosphere: A threefluid approach, J. Geophys. Res., 106 (A12), 29401-29408, 2001.

Wang, C., Richardson, J. D., and Gosling, J. T.: A numerical study of the evolution of the solar wind from Ulysses to Voyager 2, J. Geophys. Res., 105 (A2), 2337-2344, 2000. 